\title{
Discussion
}

\section{Alzheimer Research Forum Live Discussion: Non-coding RNAs in Neurodegeneration}

\author{
(http://www.alzforum.org/res/for/journal/transcript.asp?liveID=141)
}

Participants: Ben Albensi (St. Boniface Research Center/University of Manitoba), Gail Breen (University of Texas at Dallas), Anil Cashikar (Medical College of Georgia), Xiyun Chai (Eli Lilly and Company), Raymond Chang (The University of Hong Kong), Trinna Cuellar (University of California at San Francisco), Peter Frederikse (UMDNJ-New Jersey Medical School), Mansuo Hayashi (Merck \& Company, Inc.), Sebastien Hebert (KULeuven and VIB), June Kinoshita (Alzheimer Research Forum), Kenneth Kosik (University of California, Santa Barbara), Justin Long (Indiana University School of Medicine), Bryan Maloney (Indiana University School of Medicine), Lisa Mizumoto (Hoag Memorial Hospital Presbyterian), Virgil Muresan (UMDNJ-New Jersey Medical School), Joel Neilson (MIT Center for Cancer Research), Peter Nelson (University of Kentucky), Rodney Perry (University of Alabama at Birmingham), Neil Smalheiser (University of Illinois at Chicago), Lisa Stanek (Massachusetts General Hospital), Matthew Townsend (Merck \& Company, Inc.), Laura Turner (Eisai London Research Laboratories), Samuil Umansky (Xenomics Inc.), Wang-Xia Wang (University of Kentucky), Guilian Xu (University of Florida).

Note: Transcript has been edited for clarity and accuracy.

Peter Nelson: I am interested in brain microRNAs (miRNA). I have been interested that in both my experience, and in anecdotal stories from others, miRNA profiling differs somewhat across different platforms. Any comments?

Neil Smalheiser: Could you elaborate?

Joel Neilson: My own experience has been that miRNA expression platforms give somewhat differing results.

Peter Nelson: To elaborate. . I have found that the results of microarrays can be different from each other, and that Northern blots can be somewhat different, too. I have heard other people with the same issues. I think it gets to the fact that we need a bit more technical "attention to detail"; there has been a boon in miRNA pro- filing platforms with little attention to differences between them, as well as technical details such as labeling and so forth.

Peter Nelson: By analogy, the earlier results with messenger RNA (mRNA)-related microarrays were somewhat hard to replicate; it was not until years went by and the whole field acknowledged that "its" credibility was in jeopardy, that people made more rigorous standards and got more replicable results.

Anil Cashikar: I think profiling may be a better choice than expression levels. Perhaps one does not need too much of these RNAs for them to do their job.

Benedict C. Albensi: So how much is enough, then?

Peter Nelson: Anil, my impression, which may well be wrong, is that one needs a fair amount of an miRNA 
to do anything if it is not acting as a "slicer," because it needs to physically inhabit given target mRNAs to do its job...?

Sebastien Hebert: Peter, in our experience, the miRNA profiling data from human brain needed to be backed up by (at least) quantitative real-time polymerase chain reaction (qRT-PCR) in order to discriminate among miRNA family members. Overall, the profiling gave us a good hint but needed to be independently confirmed.

Neil Smalheiser: I have used two different platforms from the same company, and there were lots of differences related to design and inclusion of controls, but not so much difference in actual intensity values.

Joel Neilson: It is very difficult to say one way is better than another. However, some things to consider are as follows: direct labeling of microRNAs using something like a ligase is dependent upon the 3' sequence of the RNA, and unlike DNA ligases, RNA ligases have a sequence specificity. If using this method, I think that it is important to compare two samples (e.g., cy3/cy5), because then the relative efficiency of labeling washes out.

Neil Smalheiser: The biggest variable seems to be the way that people analyze, cleanse, normalize, and compare the data.

Joel Neilson: I agree with both Sebastien's assertion that discrimination is important, and also Neil's assertion that analysis plays a large role in array analysis.

Neil Smalheiser: Certainly if you compare two samples on the same chip, you have paired data that are more reliable than comparing two different chips. You also need internal controls, that I am not sure are routine.

Peter Nelson: I think that the miRNA profiling experiments are quantumly different from mRNA studies, though; there is something like 100-fold difference in the numbers of expressed genes. In this context, the "controls" referrent to statistical studies of mRNAs are, I think, just different. I think of an miRNA profiling experiment as more analogous to a big Northern blot than a small Affymetrix array.

Anil Cashikar: It is also possible that some miRNAs may actually regulate "other" RNAs and thus complicate the analysis.
Sebastien Hebert: We used the Ambion V2 platform where Assuragen analyzed the data. From our microarray facility, these platforms were not the most sensitive, however.

Kenneth Kosik: With regard to profiling, I have been most impressed with the RT-PCR multiplex methods because the dynamic range is greater than the array bases methods. The biggest issue with the RT-PCR multiplex is the stratospheric cost. The costs are more reasonable if you have a candidate and then do single plex.

Peter Nelson: I can only say about PCR, having done some, I get a bit chaffed when people stick in that "quantitative term" as though it were terribly accurate. I am not saying it is not broadly quantitative, but at least in my hands I would say often not.

Neil Smalheiser: By the way, we are switching to multiplex RT-PCR instead of microarrays when possible.

Kenneth Kosik: The AB system allows one to distinguish miRNA family members-single base discriminartion-in their single plex but not the multiplex that they have commercialized.

Anil Cashikar: Comment on cost: I guess a central platform for non-coding RNA (ncRNA) should be developed (by NIH?) where several disease models may be screened.

Benedict C. Albensi: Yes, could someone mention some of the specific costs?

Peter Nelson: I have heard quotes in the $\$ 500-\$ 900$ range per profiling experiment. Guiliang Tang down here at the University of Kentucky has a nice array that is pretty cheap in-house (about half of "commercial" arrays).

Joel Neilson: Exiqon goes roughly $\$ 800$ per array including bioanalyzer, labeling of the two samples, hybridization, and data processing and analysis by the company. You just send them RNA. I hesitate to throw this out there because of my own involvement with it, but I was just involved in a collaboration with a group at the Harvard CBR Institute for Biomedical Research (Haoquan Wu and Manju Swamy) that published sideby-side cloning, array, and qRT-PCR data of CD8 lymphocytes in PLOS ONE [1]. For people involved in tak- 
ing a look at direct comparisons using different techniques, this might be a reasonable resource.

Kenneth Kosik: Once finding different miRNA expression patterns in human brain, the follow-up mechanistic experiments are difficult.

Anil Cashikar: I agree with Kenneth Kosik: If more than 50 percent of the transcripts are not translated, then are we missing a really big step in biology?

Peter Nelson: $\ldots$ and what about "biological activity"? As Ken and others have indicated, maybe some "expressed" miRNAs are sequestered and are thus analogous to non-translated mRNAs?

Anil Cashikar: I mean something in the order of transcription, translation, etc.

Trinna Cuellar: I, too, have experienced varying results among miRNA arrays, Northern blotting, and Taqman ${ }^{\circledR}$ qRT-PCR. One thing we have wondered is if some of the miRNAs we are studying are undergoing post-transcriptional modifications, such as 3' end modifications or editing, altering their known mature sequence, which may affect the results, depending on probe sequence and stringency. Another contributing factor may be coexpression of miRNA family members. The Taqman probes may be more stringent than our miRNA array probes, thus contributing to the differences in results.

Peter Nelson: Great points, Trinna.

Anil Cashikar: Comment on disease mechanisms: the ncRNAs certainly open new possibilities for disease mechanisms, but it appears to add the oxidative damage/protein misfolding, etc. Any comments, Dr. Nelson?

Peter Nelson: Anil, I think it is fair to say it is a wideopen field. It is a challenge to adapt "old" ideas and techniques.

Joel Neilson: To me, one of the most interesting areas of this field right now is post-transcriptional miRNA maturational regulation.

Trinna Cuellar: Joel, I agree with you. Some of the papers published in the last year or so demonstrating post-transcriptional regulation of miRNAs are fascinating.
Peter Nelson: ... and on the post-transcriptional processing tip, we have all seen on Northerns how some miRNAs have a different percentage of pre-miRNA to mature miRNAs.

Kenneth Kosik: The kinetic relationship between premiRNA and mature miRNA is quite interesting. I think the regulatory step is the generation of the mature miRNA in the RISC.

Neil Smalheiser: I agree with Ken, except that I think the conversion from pre-miRNA to miRNA may occur, at least in part, near the synapse and not in the cell body.

Kenneth Kosik: Yes, thanks to your work, Neil, we know the machinery is out in the dendrites to effect this maturation step.

Peter Nelson: Ken, could you comment about how different miRNAs are processed to different neuronal compartments? This is a totally fascinating area.

Kenneth Kosik: Peter, that would be a lecture in itself. But we, too, are very interested in this facet.

Trinna Cuellar: Neil, I think that is a fascinating concept of local miRNA maturation near the synapse. I have often wondered how an miRNA is transported to the synapse and if there are cis-acting elements within the pre-miRNA or mature miRNA directing them there. Do you have any ideas on this?

Neil Smalheiser: I have not studied that in detail, but favor the idea that the routing is due to proteins that bind and transport them, not necessarily sequence-specific for individual pre-miRNAs or miRNAs.

Kenneth Kosik: Yes, the specificity is conferred by the miRNA and the RISC machinery is more general. Processing bodies ( $\mathrm{P}$ bodies) also have a role.

Peter Nelson: As you have mentioned, it just seems as though the ultra-geometrically asymmetric neurons would have a "vested interest" in adapting locally.

June Kinoshita: I was wondering if anyone is interested in learning more about the microRNA sponge concept from Neilson and Ebert. Any questions or interest in trying it in your laboratory?

Peter Nelson: I am interested. As Ken said, figuring out mechanism in tissue culture after finding miRNA changes by profiling is deceptively hard. 
Neil Smalheiser: Depends on what the changes are, whether they can be interpreted easily or not. If the miRNA fit a pattern, that is better than simply isolated changes up or down.

Joel Neilson: I am actually really curious if anyone has been able to employ this for either miRNA loss of function (LOF) in neurons or as a tracker for miRNAmediated asymmetric delivery of mRNAs in neurons.

Kenneth Kosik: Joel, we are doing this and should know soon if it is working.

Sebastien Hebert: Before time runs out, I would like to know whether someone has interesting data to share about Alzheimer's disease, Parkinson's disease, or other neurodegenerative diseases. From our side, we have some data indicating changes in miRNA expression profiles from sporadic AD brains. As well, several affected miRNAs can regulate genes involved in the amyloid cascade. What could be the role of miRNA pathways in sporadic diseases?

Peter Nelson: Sebastien, I am barking up that same tree... which I think will be a fertile field for many investigators. I feel a bit constrained as the data are not published yet.

Sebastien Hebert: I understand, of course. We have been trying to publish our story as well, but it always comes back with criticism that we need some in vivo data (i.e., miRNA knockout mouse). Of course, not an easy road to cross.

Peter Nelson: It is becoming a bit of a recurring theme I have noticed: miRNA knockout mice. Human data are "phenomenological," but mice data are "mechanistic." My interpretation: We now have a load of phenomenological data about what happens when you mess with a mouse genome, and how far has it gotten us beyond the human data? Not that far. Not that transgenic mice are not important.
Sebastien Hebert: Well, what I can say is that out of affected miRNAs in sporadic AD, most of them are predicted to target $\mathrm{A} \beta \mathrm{PP}$ and BACE1. These observations were backed up with correlation data in patients and in mouse and in vitro using several systems.

Peter Nelson: Sebastien, our results are compatible with what you just wrote. Let us just take BACE1. I think that it will turn out to be regulated in a great many ways-transcriptional, splicing, translational, post-translational (e.g., phosphorylation)-all mixed up in space and time. And, I think, miRNAs are one avenue, too.

Joel Neilson: Sebastien and Peter, do you have a comment on the interplay between miRNAs and the stress response and these pathologies that you would be willing to share?

Peter Nelson: I am not exactly sure about the stress response, as it is not monolithic (as you know, many miRNAs act differently under different stresses). As with other things, I think that the effects of details are important: patient details, pathology details, details about sampling (gray/white matter), details about what the patients were taking, postmortem intervals, etc. It is a real challenge to sort through.

June Kinoshita: Our time is formally at an end. Thank you, everyone, for participating.

\section{Reference}

[1] Wu H, Neilson JR, Kumar P, Manocha M, Shankar P, Sharp PA, Manjunath N (2007) miRNA Profiling of Naïve, Effector and Memory CD8 T Cells. PLoS ONE 2, e1020. 